\title{
Inclusão Digital na Terceira Idade: Considerações sobre a Experiência com a Informática
}

\author{
Felipe T. Guimarães ${ }^{1}$, Giani Carla Ito ${ }^{2}$, Mayara Cristina Pereira Yamanoe $^{3}$ \\ ${ }^{1}$ Universidade Tecnológica Federal do Paraná (UTFPR) \\ Cornélio Procópio - PR - Brasil \\ ${ }^{2}$ Universidade Tecnológica Federal do Paraná (UTFPR) \\ Santa Helena - PR - Brasil \\ ${ }^{3}$ Universidade Tecnológica Federal do Paraná (UTFPR) \\ Francisco Beltrão - PR - Brasil \\ felipegealunos.utfpr.edu.br \\ \{mayarayamanoe, gianiito\}@.utfpr.edu.br
}

\begin{abstract}
Technology, as it grows and expands, transforms culture, and so does society and the way people live, for they are the results of one's own social relations. At the same time that these changes impact society, there are social groups that are excluded digitally, as is the reality of some elderly people. This article presents the Digital Inclusion of a group of elderly people, taught by the author and promoted by the Open University to the Third Age, in partnership with the Federal Technological University of Paraná. From this, the objective of this article is to analyze and discuss the impact of Digital Inclusion on the life of the elderly person. The results indicated that Digital Inclusion can positively impact the elderly, both at educational and health levels.
\end{abstract}

Resumo. A tecnologia, conforme cresce e se expande, transforma a cultura, e assim a sociedade e a maneira como as pessoas vivem, pois são resultados das próprias relações sociais. Ao mesmo tempo que estas mudanças impactam a sociedade, existem grupos sociais excluídos digitalmente, como é a realidade de alguns idosos. Este artigo apresenta a Inclusão Digital de um grupo de idosos, ministrada pelo autor e promovida pela Universidade Aberta à Terceira Idade, em parceria com a Universidade Tecnológica Federal do Paraná. A partir disso, o objetivo deste artigo é analisar e discutir o impacto da Inclusão Digital na vida da pessoa idosa. Os resultados indicaram que a Inclusão Digital pode impactar positivamente os idosos, tanto em níveis educativos quanto de saúde.

\section{Introdução}

A sociedade contemporânea imprime maior valor para a informação que se difunde de forma rápida e intensa por meio das diversas Tecnologias Digitais de Informação e Comunicação (TDIC), tais como o celular, computador, televisão, aplicativos e entre outras. A habilidade de grupos e indivíduos em acessar e utilizar as TDIC é chamada de Inclusão Digital, englobando o acesso a internet, apropriação de hardware e software, bem como serviços e informações relevantes que levam ao desenvolvimento de habilidades para alfabetização digital necessárias para o uso efetivo destas tecnologias. 
VIII Congresso Brasileiro de Informática na Educação (CBIE 2019)

Anais do XXV Workshop de Informática na Escola (WIE 2019)

Atualmente, é possível identificar que as pessoas idosas também têm buscado a apropriação de tecnologias que as assegurem a conectividade por meio de internet ou dados móveis em seu dia a dia. Segundo [Becker et al. 2012], o custo da exclusão digital é alto, pois quando não incluído digitalmente, a participação do indivíduo, na maior parte dos aspectos da sociedade moderna ocidental, está comprometida, considerando desde questões relacionadas ao campo econômico, passando pelas educacionais até o acesso à saúde e o engajamento cívico. No mesmo sentido do não acesso à cultura letrada por meio do alfabetismo, problemática histórica que tem sido dirimida no decorrer do tempo, o analfabetismo tecnológico também constrói barreiras para o acesso à direitos. Para além disso, um acesso não orientado à apropriação do uso dos artefatos tecnológicos de forma adequada pode ser um falseamento da inclusão, colocando em risco o usuário.

A Organização Mundial da Saúde (OMS) classifica a população idosa entre aqueles que possuem idade igual ou superior a 60 anos. Mesmo que aparente precoce essa classificação para esta idade se considerarmos os países em desenvolvimento, a OMS recomenda às autoridades a desenvolverem políticas sociais baseadas não somente na idade cronológica, mas também na idade biológica, pois, apesar de possuir a mesma idade, as pessoas apresentam variações na saúde, em participação na sociedade e em níveis de independência [Organization et al. 2002].

A projeção do Instituto Brasileiro de Geografia e Estatística [IBGE 2018], sobre o envelhecimento da população brasileira, evidencia que em dez anos, considerando 2019, a população de pessoas com 60 anos, ou mais, de idade atingirá 40.935 .475 milhões, diferença de aproximadamente $28 \%$ a mais sobre o número de idosos que existe atualmente. Dados estes que evidenciam que, apesar de os idosos do futuro serem pessoas que provavelmente já terão acesso à tecnologia em idade anterior, ainda teremos um público resquício de idosos que demandará pela apropriação tecnológica e, principalmente, maneiras de aprender a como usá-la. No entanto, é válido destacar que essa é uma problemática contemporânea e muito atual, que deve conjugar ações de inclusão digital para todos os públicos, convergindo para a construção de condições reais de acesso tanto aos nativos digitais quanto aos migrantes.

É importante salientar que os processos de inclusão digital devem considerar as especificidades do público idoso. Sabe-se que o envelhecimento compromete, de forma até a limitar, a ação cognitiva do ser humano, ou seja, pode frear o processo de aquisição do conhecimento. Para [Nunes et al. 1999], existe um declínio da Atenção Seletiva e da Atenção Dividida na mente dos idosos. Atenção Seletiva refere-se à capacidade de discernir informações importantes, já a Atenção Dividida, à capacidade de processar duas ou mais informações ao mesmo tempo. Com isso, conclui-se que o envelhecimento é responsável pelo comprometimento da memória e todo seu sistema de armazenamento, sistema este que inclui: memória sensorial, memória de curto prazo e a memória de longo termo [Kreis et al. 2007].

Em contrapartida, existem possibilidades para que os idosos possam retomar, e inclusive manter, memórias, ao mesmo tempo em que são estimuladas atividades cognitivas no cérebro. Uma destas possibilidades é a Inclusão Digital. O constante estímulo das atividades cognitivas pode proporcionar bem-estar ao idoso, sensação de valor e utilidade [Reis 2018], inclusão social e participação em grupos e comunidades virtuais, tais como redes sociais, jogos digitais, e-commerce, e entre outras atividades que até pouco tempo atrás 
VIII Congresso Brasileiro de Informática na Educação (CBIE 2019)

Anais do XXV Workshop de Informática na Escola (WIE 2019)

eram incomuns para a terceira idade. Tudo isso pode contribuir com o desenvolvimento cognitivo do idoso, melhorando sua saúde mental e garantindo ganhos para a plasticidade cerebral.

Diante do contexto exposto, o objetivo desse trabalho foi analisar o impacto da Inclusão Digital na qualidade de vida do idoso, por meio da disciplina Inclusão Digital, do curso de Ciências do Envelhecimento Humano da Universidade Aberta à Terceira Idade (UNATI), ofertado pela Universidade Estadual do Oeste do Paraná (UNIOESTE), Campus de Francisco Beltrão, no Estado do Paraná, e obteve a cooperação do curso de Licenciatura em Informática, ofertado pela Universidade Tecnológica Federal do Paraná (UTFPR), Campus de Francisco Beltrão, no Estado do Paraná. Desse modo, para auxiliar o leitor na compreensão do texto, inicialmente será apresentada a UNATI, assim como os seus principais objetivos.

\section{A Universidade Aberta à Terceira Idade - UNATI e a ampliação ao acesso à Educação pelos Idosos}

A influência da cultura digital na sociedade é um fato a ser considerado, e está relacionada à quantidade, crescente, de pessoas que acessam as TDIC por meio de dispositivos móveis [Pereira et al. 2018].

Apesar da inserção de artefatos tecnológicos no cotidiano já serem realidade a partir da Revolução Industrial, as tecnologias da informação e comunicação são recentes e sua popularização teve início nas últimas duas décadas. Sendo assim, a terceira idade tem dificuldade de compreender e se apropriar da informática, já que essa é incorporada às práticas sociais em contextos específicos e, especialmente na realidade brasileira, não foi democratizado desde o início. E mesmo estando inseridos na sociedade, como consumidores ou força produtiva, os idosos tendem a sofrer preconceito pelo espectro idade, o que não é diferente quando tratamos de questões tecnológicas, muito pelo contrário.

Uma pesquisa, conduzida por [Dantas et al. 2014], no Instituto Federal do Rio Grande do Norte (IFRN), conclui que pessoas da terceira idade sofrem preconceito por não saber utilizar a tecnologia, ao mesmo tempo que não conseguem aprender pela falta de cursos de informática voltados a esta faixa etária. Apesar da pesquisa levantar a questão em uma região, não é desacertado considerar que isso se reflete de forma quase geral pelo país.

É também considerando o sentido de ampliação ao acesso à educação de forma geral, que em 1993 foi inaugurada a Universidade Aberta da Terceira Idade (UNATI), na Universidade do Estado do Rio de Janeiro (UFRJ) [UERJ 2019], que inicialmente era vinculada ao Instituto de Medicina Social (IMS) da UFRJ, sendo este feito um projeto do Professor Américo Piquet Carneiro [Furtado and da Silva 2008]. As ações da UNATI perpassam ensino, pesquisa e extensão voltados à população idosa, buscando contribuir com as políticas de assistência específica a esse público e, um de seus objetivos centrais era: "Promover cursos/oficinas para pessoas idosas, visando atualizar seus conhecimentos e integrando-os à sociedade contemporânea" [UERJ 2019].

Atualmente, a UNATI é um curso-programa, vinculado a atividades de extensão universitária, para atender, exclusivamente, a terceira idade, e já se espalhou em universidades de todo o país. O requisito idade é necessário para ser candidato a uma vaga no curso 
VIII Congresso Brasileiro de Informática na Educação (CBIE 2019)

Anais do XXV Workshop de Informática na Escola (WIE 2019)

da UNATI. Na UNIOESTE de Francisco Beltrão, é exigida idade mínima de 50 anos para participar. Já no campus dessa mesma universidade, em Cascavel, a idade mínima é de 55 anos. A idade pode variar de acordo com a região e a universidade que está ofertando o curso. As disciplinas são ministradas pelo corpo docente e discente da universidade, de forma gratuita, sendo este o compromisso da universidade com a sociedade. O objetivo da UNATI, desde sua criação, é ser centro de ensino, pesquisa e extensão que giram em torno do envelhecimento, dando visibilidade para os idosos, ao mesmo tempo que trabalha na reinserção deles na sociedade.

\section{Inclusão Digital}

Para compreender o significado e efeitos que propõe a Inclusão Digital, esse estudo se embasará na investigação de [Marcon and Carvalho 2015]. Esses autores apresentam suas conclusões acerca de concepções sobre Inclusão Digital a partir de resultados obtidos em pesquisas de teses e dissertações. Fez-se necessário expor algumas concepções de Inclusão Digital que dialoguem com o objetivo da UNATI. Por isso, foram consideradas interpretações acerca da Inclusão Digital que levem à apropriação do tecnológico, o ensino e a atuação no meio social pela tecnologia.

[Marcon and Carvalho 2015] compreendem a inclusão digital como o processo que torna o ser humano apto a ser produtor ativo de cultura e conhecimento, privilegiando comportamentos da sociedade contemporânea como a interação e a colaboração. Assim, não apenas ter contato, a inclusão digital é também a utilização e o desenvolvimento, ela acontece enquanto está ativa. Esta conclusão compõe a inclusão digital em três eixos:

1. Apropriação/Fluência/Empoderamento tecnológico: significa ter acesso, desenvolver conhecimento necessário para utilizar, interagir e entender a tecnologia, até que possa ser independente no uso dela;

2. Produção/Autoria individual/coletiva de conhecimento e de cultura: consiste em ser produtor de conteúdos e transmiti-los, interagir com outros sujeitos, por meio da tecnologia superando os limites espaciais e temporais;

3. Exercício da cidadania na rede: é a garantia da diversidade estar inserida no espaço, e transformar a si e outros por meio da tecnologia, dialogando com um interesse em particular ou coletivo.

Diante desta pesquisa evidencia-se que a Inclusão Digital visa, além da apropriação da tecnologia, o desenvolvimento de habilidades que possam modificar a realidade dos sujeitos que dela desfrutam. Dessa forma, a Inclusão Digital consegue se articular aos propósitos de ampliação do desenvolvimento bio-psíquico-social de idosos, sendo válida e justificável para alcançar os objetivos com os quais a UNATI trabalha.

Ao envelhecer, os idosos passam a dispor de um pouco mais de tempo, na maioria dos casos eles estão aposentados ou trabalhando menos. Este tempo pode ser ressignificado, sendo destinado à atividades prazerosas, ao mesmo tempo que podem ter também um caráter pedagógico. Por meio da Inclusão Digital, os idosos adquirem a oportunidade de aprender sobre informática, tecnologia, desenvolver novas habilidades relacionadas às TIC e ao mesmo tempo aprimorar sua saúde mental e emocional: "O domínio das tecnologias computacionais amplia experiências, amizades e horizontes e proporciona uma forma de lazer segura e desafiadora, evitando doenças tal como a depressão" [Marcon and Carvalho 2015]. 
VIII Congresso Brasileiro de Informática na Educação (CBIE 2019)

Anais do XXV Workshop de Informática na Escola (WIE 2019)

\section{Materiais e Métodos}

A abordagem metodológica utilizada neste trabalho foi qualitativa, seguida do método exploratório e descritivo das informações. Assim, foi considerado o perfil sociodemográfico dos alunos e seu nível de conhecimento tecnológico antes de iniciar o curso, para inferir na análise sobre o questionário aplicado ao fim do mesmo.

Para a coleta dos dados, foi utilizado de um questionário online desenvolvido com a ferramenta Formulários Google [Google 2019], demonstrado pela Figura 1, e aplicado ao final do trimestre para os alunos do curso de Ciência do Envelhecimento Humano, na disciplina de Inclusão Digital. O Formulários Google facilita a interpretação dos dados, uma vez que a ferramenta realiza automaticamente a tabulação dos dados, gerando gráficos e tabelas das respostas obtidas.

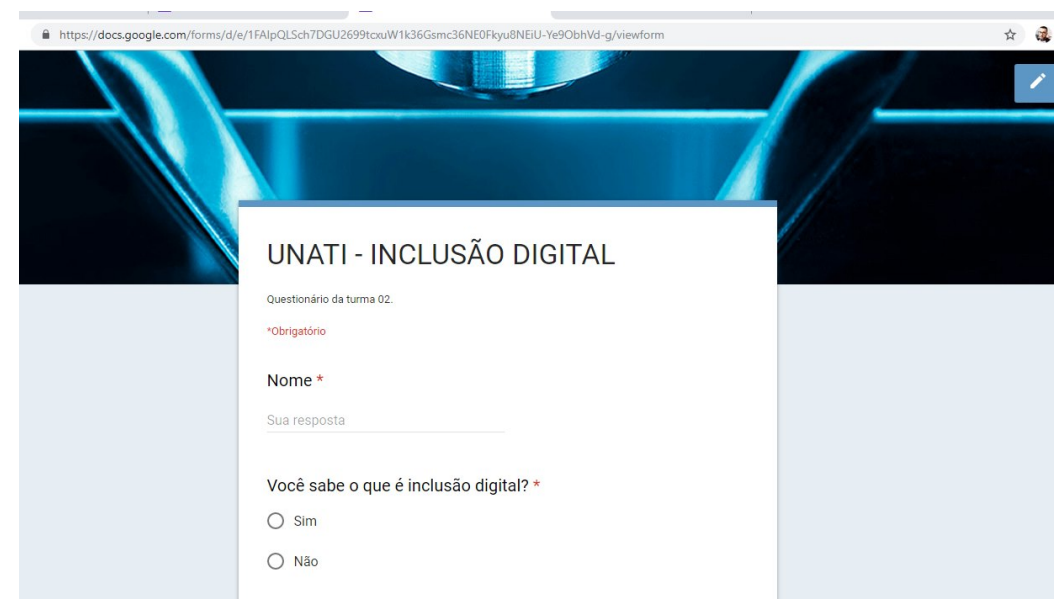

Figura 1. Questionário aplicado ao final do curso

Os métodos de ensino escolhidos, para a disciplina de Inclusão Digital, foram Aulas Expositivas e Dialogadas e a Sala de Aula Invertida. Essas metodologias foram selecionadas por serem metodologias de ensino ativas, o que amplia as possibilidades de tornar o processo de aprendizagem significativo, ao passo que proporciona mais autonomia para o aluno. Nas metodologias ativas, exige-se do aluno um comportamento ativo durante o processo da construção de conhecimento, bem como podem viabilizar a utilização da Informática como instrumento pedagógico [Guimarães et al. 2018]. Assim, a Informática pode ser utilizada como meio, mas também como fim. No âmbito da Inclusão Digital faz-se necessário aprender o que são e como utilizar as ferramentas tecnológicas, além de inseri-las como mediação para produção do conhecimento.

Foram matriculados 38 alunos na disciplina de Inclusão Digital, dos quais 28 alunos permaneceram até o final do curso. Dessa forma, foram esses 28 alunos que contribuíram com o questionário aplicado ao fim do curso. As perguntas formuladas para os alunos podem ser conferidas na Tabela 1:

As aulas de Inclusão Digital foram ministradas pelo autor do trabalho durante dois trimestres de 2018 na UNIOESTE, no campus de Francisco Beltrão. No decorrer da disciplina, trabalhou-se com os alunos conceitos sobre a Inclusão Digital, o que são tecnologias digitais, bem como a relação destas com as mudanças de comportamentos sociais, Informática básica, Internet e o uso de smartphones. Os alunos participaram de 
VIII Congresso Brasileiro de Informática na Educação (CBIE 2019)

Anais do XXV Workshop de Informática na Escola (WIE 2019)

Tabela 1. Perguntas contidas no questionário

\begin{tabular}{r|l} 
Número & Pergunta \\
\hline P. 01 & Você sabe o que é Inclusão Digital? \\
P. 02 & Na sua opinião, a informática melhorou a sua vida? Explique. \\
P. 03 & O que sua cidade precisa fazer para facilitar o uso de tecnologias por idosos? \\
P. 04 & Na sua opinião, é importante a tecnologia ser acessível?
\end{tabular}

aulas teóricas e práticas com seus próprios celulares e os computadores do laboratório da Universidade. Como forma de incentivá-los a utilizar e incorporar a tecnologia no uso diário, foram criados meios para comunicação entre os alunos e o professor em redes sociais e $e$-mail.

O questionário proposto para este trabalho teve como objetivo analisar se os idosos entendiam as tecnologias e de que forma, para eles, se houve contribuição a partir do uso de tecnologias para melhoria na qualidade de vida e bem-estar social.

\section{Resultados e Discussão}

Primeiramente será apresentado o perfil sociodemográfico dos idosos participantes deste estudo, e em seguida será apresentada a análise dos idosos sobre a Inclusão Digital em sua vida. Para tanto, os 28 alunos participantes foram classificados como A01 até A28.

Dos alunos participantes desta pesquisa, 22 são do sexo feminino e apenas 6 do sexo masculino. A Figura 2 mostra o nível de escolaridade dos alunos da UNATI.

\section{Escolaridade:}

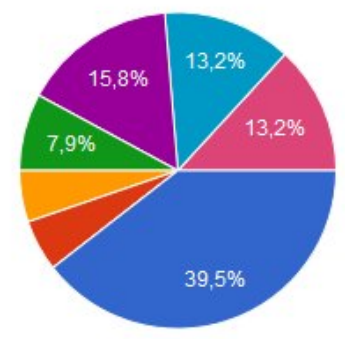

Ensino Médio

Superior Completo

- Especialização

Superior Incompleto

- Ensino Fundamenta

Ensino Fundamental Incompleto

- Ensino Médio Incompleto

Figura 2. Nível de escolaridade do público pesquisado

Com o objetivo de compreender se a Inclusão Digital impactou positivamente a vida do idoso, é apresentada a seguir a análise das respostas obtidas por meio do questionário aplicado ao final do curso.

Sobre a primeira questão P.01 (Tabela 1), mostra que 100\% dos alunos responderam "Sim", conforme mostra a Figura 3, afirmando ter aprendido o conceito de Inclusão Digital. A primeira pergunta foi pensada levando em consideração a primeira aula da disciplina, na qual evidenciou-se que não conheciam o significado de Inclusão Digital. Considerando o nível de escolaridade dos alunos e o longo período passado desde que eles 
VIII Congresso Brasileiro de Informática na Educação (CBIE 2019)

Anais do XXV Workshop de Informática na Escola (WIE 2019)

frequentaram alguma modalidade de ensino, pode-se inferir que não tiveram a oportunidade de aprender conceitos atuais da tecnologia, principalmente conceitos que não dialogam com sua realidade. Porém, atualmente os alunos sabem explicar o que é a Inclusão Digital.

Já a segunda questão, P.02, foi elaborada pensando em investigar os pontos positivos da Inclusão Digital se tornando realidade na vida do idoso. Notabilizou-se a satisfação dos alunos em relação a tecnologia, principalmente no que concerne a um bem-estar social proporcionado pelo uso do computador ou celular. A sensação de bem-estar e alegria pôde ser corroborado por meio de algumas respostas obtidas da questão P. 02 (Tabela 1), transcritas na Tabela 2.

\section{Você sabe o que é inclusão digital? \\ 28 respostas}

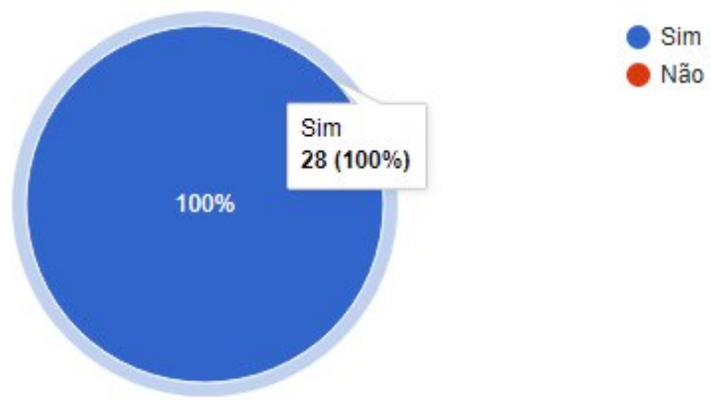

Figura 3. Dados da primeira pergunta do questionário

Tabela 2. Dados obtidos por meio do questionário

Aluno $\mid$ Resposta

A01 "Sim [...] olho receitas na internet, estudo e posso falar com meu filho [...]";

A05 "Sim. Eu decidi procurar o curso pra sair um pouco de casa e aprender [...]";

A12 "Melhorou. Eu queria usar o celular para tirar fotos e enviar meu trabalho [...]";

A13 "Bastante, eu não terminei escola, nunca pensei em eu estudar em uma universidade.";

A19 "Sim. Eu comprei meu celular depois que comecei o curso aqui [...];

A21 "Sim, eu morria de medo de usar internet, minhas filhas brigavam [...]";

A26 "Sim, no meu trabalho era obrigado a usar o computador, ajudou nisso".

Um dos alunos denominou a tecnologia como um "remédio" para a solidão, outro como a "solução" para independência. É possível encontrar algumas respostas indicando que alguns dos idosos moram sozinhos e/ou longe de seus filhos, porém o acesso a internet fez com que eles estivessem mais presentes. Apesar de inúmeras explicações diferentes, todos os alunos concordam que a Inclusão Digital melhorou algo em sua vida.

Evidentemente, questões mais complexas em torno das concepções de tecnologia não puderam ser trabalhadas, já que se tratava de um processo inicial de inclusão a que 
VIII Congresso Brasileiro de Informática na Educação (CBIE 2019)

Anais do XXV Workshop de Informática na Escola (WIE 2019)

demandava um enfoque funcional da tecnologia. Entende-se, no entanto, a partir de perspectivas críticas, que não se pode atribuir um caráter salvacionista e totalizante à tecnologia, mas que ela precisa ser compreendida nas relações em que se insere. Nesse caso, em específico, o papel de ampliação das possibilidades de conexão são, sem dúvidas, uma positividade no contexto dos alunos idosos.

A terceira questão, P.03 (Tabela 1), buscava identificar se os alunos sentiam-se injustiçados ou isolados na cidade, em que habitam, pelo simples fato de serem idosos. Muitas discussões foram levantadas pelos alunos, alguns responderam que a solução está nas mãos de políticos: "Precisa de mais cobrança das entidades públicas e cursos para terceira idade", "precisa pedir políticas para apoio do governo". Também foram levantadas questões econômicas: "preços mais baixos em aparelhos eletrônicos" e "precisa investir mais em cursos e ensino para o idoso e dar segurança", o que cabe a pensar questões sobre atenção e incentivo na educação do idoso.

Na cidade de Francisco Beltrão não existem escolas técnicas de informática ou cursos específicos de tecnologia para terceira idade, de forma gratuita ou paga. Muitos alunos responderam que gostariam de encontrar mais cursos relacionados a tecnologia e informática. Emerge da prática social, portanto, uma relação complexa entre tecnologia e sociedade, especialmente em seus desdobramentos políticos e econômicos, que é percebida pelos sujeitos. Nessa relação, encontra-se a exclusão digital e como ela se constitui de diferentes maneiras, sendo, nesse caso, marcada pela especificidade geracional.

Por fim, a última questão P.04 (Tabela 1), teve o objetivo de mensurar o quão importante era a acessibilidade tecnológica para o idoso. Conforme demonstra a Figura 4, 89\% dos alunos concordam que é importante a tecnologia ser acessível. Este resultado contraria a expectativa do professor sobre a questão, uma vez que a Inclusão Digital ocorre mais facilmente quando existe mais acesso às tecnologias. Em contrapartida é compreensível que alguns alunos podem não ter entendido a acessibilidade como uma necessidade de todos, ou algo que contribua para os idosos aderirem aos artefatos tecnológicos.

Na sua opinião, é importante a tecnologia ser acessível?

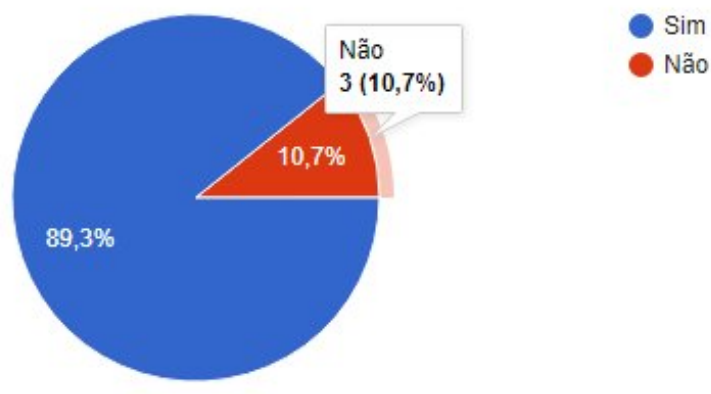

Figura 4. Dados da última pergunta do questionário 
VIII Congresso Brasileiro de Informática na Educação (CBIE 2019)

Anais do XXV Workshop de Informática na Escola (WIE 2019)

A UNATI criou, além de colegas de curso, uma comunidade para os idosos. Eles construíram relações de amizade e passaram a interagir muito entre si, por meio de aplicativos como o WhatsApp e o Facebook, que também foram instrumentos utilizados em sala de aula. Durante o curso, alguns alunos relataram satisfação em aprender a utilizar aplicativos de banco pelo smartphone, e assim não precisarem mais sair de casa e enfrentar filas nos caixas eletrônicos. Esses aspectos revelam como o apoio do artefato tecnológico pode indicar um bem a saúde, bem como a conquista de maior independência, segurança e comodidade.

\section{Considerações Finais}

A presente pesquisa objetivou analisar aspectos que poderiam causar impacto positivo, na qualidade de vida, que a Inclusão Digital pode trazer para a vida da pessoa idosa.

Durante o processo do curso realizado observou-se que, aos poucos, os alunos entendiam a proposta da disciplina e, em seu ritmo, os idosos encontraram alguns caminhos para ampliarem seu conhecimento. Com isso, ao final do curso os avanços que os alunos alcançaram foram em níveis satisfatórios para o professor. Identificou-se também que, de acordo com os resultados da pesquisa, a Inclusão Digital pode causar impactos positivos na vida do idoso, e em diferentes aspectos, como na aproximação com a família, no enfrentamento ao sentimento de tristeza e solidão e na independência para utilização de seus artefatos próprios, como o celular.

Os resultados obtidos a partir da análise dos dados foram satisfatórios, bem como superou as expectativas do pesquisador. Além disso, a partir da UNATI, os idosos puderam ter uma experiência inédita, pois alguns alunos nunca haviam tido contato com um computador em toda a sua vida. Assim, pode-se considerar que estes alunos estão incluídos digitalmente. Mas, e o que isso significa? Significa que, é possível incluir pessoas idosas no contexto digital, e auxiliá-las no desenvolvimento de sua independência na cultura digital.

A terceira idade não está "velha demais" para aprender. Pelo contrário, é uma geração que possui muito interesse e, como todos sujeitos, independente de idade, pode se dedicar a aprender o que deseja. se viabilizadas as condições para o processo de ensino-aprendizagem. Entender as especificidades para a educação de adultos e idosos é fundamental para que os resultados de um trabalho como esse sejam positivos. Diante disso, considera-se importante ampliar os estudos sobre essa relação entre ensino de tecnologia e questões geracionais, bem como contribuir com metodologias que considerem as particularidades desse público.

Geralmente, um jovem busca a universidade com objetivo de galgar espaço no mundo do trabalho. Já os alunos da UNATI buscaram a universidade para melhorar a qualidade de vida, estudar e encontrar um grupo social que não os exclua. Em conclusão, essa pesquisa contribui com um olhar mais realista, ao mesmo tempo que sensível, sobre a condição do idoso na sociedade atual, levando a repensar possibilidades de transformar a informática em ferramenta para melhoria da vida dos idosos, o que inclui o acesso à educação de qualidade e à inclusão digital. 
VIII Congresso Brasileiro de Informática na Educação (CBIE 2019)

Anais do XXV Workshop de Informática na Escola (WIE 2019)

\section{Referências}

Becker, S., Crandall, M., Coward, C., Sears, R., Carlee, R., Hasbargen, K., and Ball, M. A. (2012). Building digital communities: A framework for action. Technical report, Institute of Museum and Library Services.

Dantas, M. R. N. et al. (2014). Os benefícios da inclusÃo digital na terceira idade: Relato de experiÊncia.

Furtado, S. R. S. and da Silva, N. C. (2008). Caminhos da história e da memória: a universidade aberta da terceira idade da uerj. Revista Brasileira de Geriatria e Gerontologia, 11(2):275-287.

Google (2019). Formulários google. Disponível em: https : / / www • google . com/ intl/pt-BR/forms/about/. Acesso em: 08 mar 2019.

Guimarães, F., Leite, M., Reinaldo, F., and Ito, G. (2018). Métodos ativos de ensino aliados com tecnologia para a prática de ensino: um relato de experiência. In Anais do Workshop de Informática na Escola, volume 24, page 333.

IBGE (2018). Projeção da população por sexo e idades simples. Technical report, Instituto Brasileiro de Geografia e Estatística.

Kreis, R. A., Alves, V. P., Cárdenas, C. J., and de Oliveira Karnikowski, M. G. (2007). O impacto da informática na vida do idoso. Revista Kairós: Gerontologia, 10(2).

Marcon, K. and Carvalho, M. J. S. (2015). Concepções de inclusão digital na formação inicial de educadores. In Anais do Workshop de Informática na Escola, volume 21, page 471 .

Nunes, R. C. et al. (1999). Metodologia para o ensino de informática para a terceira idade:: aplicação no cefet/sc.

Organization, W. H., of Nutrition Science, T. U. S., Policy, et al. (2002). Keep fit for life: meeting the nutritional needs of older persons. World Health Organization.

Pereira, M., Cardoso, P. F., and Reis, M. V. S. (2018). Uso de tecnologias educacionais por professores do ensino médio: Um estudo de caso. In Anais dos Workshops do Congresso Brasileiro de Informática na Educação, volume 7, page 105.

Reis, R. L. R. (2018). Benefícios da inclusão digital na vida da pessoa idosa: revisão de literatura.

UERJ (2019). Conhecendo a unati/uerj. Disponível em: http: / / www • unatiuerj . com.br/Conhecendo \\%20a\ะ20UnATI_completo.pdf. Acesso em: 13 jun 2019. 